\title{
ASSESSMENT OF TRIBAL HEALTHCARE INFRASTRUCTURE FOR DELIVERY OF MATERNAL HEALTH PROGRAM IN BALASORE DISTRICT, ODISHA, INDIA
}

\author{
Ranjit Kumar Dehury \\ School of Management Studies, University of Hyderabad, Telangana
}

Correspondence: ranjit@uohyd.ac.in

\begin{abstract}
INTRODUCTION:

There are still large number of maternal deaths in India nearing up to 50,000 deaths in a year which is one sixth of the world. Odisha (India) is also not performing well in maternal health care management despite its commitment to MDG and SDG. Odisha constitute $23 \%$ of tribal population, where the maternal mortality is very high and difficult to provide health services, particularly maternal health services. The study aims to assess provision of necessary health services for pregnant women in an inaccessible tribal pocket.
\end{abstract}

\section{METHODS:}

The study was conducted in Balasore district, Odisha (India) where a sizable population consists of various indigenous tribes. The Parijata tool was used to assess various health care facilities which was developed by UNICEF and Action Research and Training for Health (ARTH), Rajasthan. The availability of basic necessities like manpower, drugs and consumables are assessed along with prescribed clinical practices and procedures.

\section{RESULTS:}

The study found that there is lack of provisioning of health services in healthcare facilities. Both the district hospital at Balasore and sub-district hospitals lack basic services recommended by WHO for taking care of women. Further, the effort of government to implement a uniform program across the state hinders the tailoring of services for tribal pockets.

\section{CONCLUSION:}

The study provides remedial measures for improving the role and functioning of grass roots workers, integrating indigenous medicine with biomedicine, and revamping health information system to incorporate cultural features, thereby improving its utilization in the study area. The study raised critical issues about potential of maternal health program to deliver effective care of pregnant women in tribal dominated areas.

\section{KEYWORDS}

Tribal Health, National Health Policy, National Health Mission, Women Empowerment, Hospital management, Quality guidelines 
been sufficient which necessitated more efforts in achieving the global target. [13]

Maternal death is always a cause of concern for wider social development. Mothers usually take care of the family to a great extent for greater benefit of the family. If a mother dies it is not just the loss of the family but also the loss of entire society indirectly. [1] Overall, this will lead to loss of goodwill and potential for the development of society. [2] In India, maternal deaths are still at a high level and are always a point to ponder in government level meetings. [3] Besides the government report of large number of maternal deaths, there is also a lot of talk in media and social life about the death of women during pregnancy or related causes of pregnancy.

These topics are discussed in various forum of gender and women health and the remedial measures thereof. The discussion brings out social, economic and cultural causes along with imminent medical causes of death. Evidence found that the high maternal mortality ratio (MMR) is due to non-availability of obstetricians and skilled birth attendants in rural areas. $[4,5,6,7]$ In India, reports suggests that the skilled birth attendants (SBAs) help only $60 \%$ of cases in conducting deliveries as per protocol. [8] This should be $100 \%$ as per government target.

The statistics shows the depth and width of maternal deaths across India. There are still large number of maternal deaths in India nearing up to 50,000 deaths in a year which is onesixth of the world. [2] Most of the maternal deaths happen in India can be preventable with intervention of modern technology and medical sciences. [1] Half of pregnant women do not receive three antenatal check-ups (ANCs) during pregnancy in India leading to high vulnerability. The is similar to the provision of iron and folic acid supplements at least for 100 days during pregnancy. [3] There is always requirement of intersectoral coordination for the development of health. [2] The health systems usually details the inter-relationship between main actors in the health care system for provision of health care services. $[9,10,11]$ A study in the village of Chhainsa, Haryana (India) shows that there are many hindrances of social cultural factors responsible for poor maternal health outcomes. [12] Odisha is not performing well in maternal healthcare management despite its mandate in Millennium Development Goal (MDG) and Sustainable Development Goal (SDG). The SDG 3 clearly has a mandate for good health and well-being of the people. There was a global trend in reduction of MMR. The decline in India has not

The government of Odisha tries hard to minimize the maternal death and make various programs along with the central government for improvement. However, the result is always not satisfactory in provision of maternal health care services in hard to reach areas. Odisha ranks below Indian states so far as maternal mortality is concerned. Odisha is classifieds as a 'high-focus' state by the National Health Mission for its significantly high maternal mortality. This helps in keeping Odisha as a high priority state while providing fund for maternal health care in comparison to other states. All the schemes of Odisha health care mostly supported by National Health Mission (NHM) in one way or other. National Health Mission was launched by the federal government to enhance the health infrastructure, human resources and fiscal provisions for augmenting the health care delivery system at rural India. The NHM have a focus on a functional referral system, adequate fiscal provision, following a quality standard, ensuring human resource and capacity building at different levels through janani suraskhya yojna (JSY), which means to provide required support system for maternal health care. [1]To get the work done, accredited social health activists (ASHAs) are deployed in each village as grassroots level workers. The ASHAs help in changing the behaviour of women towards maternal health care program of government. [1]

The tribal population of Odisha are given the constitutional rights for protection by Article 366 (25) and 342 of the Constitution of India. The 'tribals' have unique features like: distinct cultural traits, geographically isolated habitat, general resistance to acculturation and overall backwardness according to Ministry of Tribal Affairs, Government of India. [14] There are around 600 different tribes across India, of which 66 tribes found in Odisha (Census, 2011). [14] The tribal population of Odisha is around $23 \%$ of the population where the maternal mortality is very high and difficult to provide health services, particularly maternal health services. The majority tribes in Odisha are Santhal, Bhumija, Kohla, and Khond. [14] Overall, there is problem in providing health services to the tribal communities of Odisha. Evidence also demonstrates poor healthcare infrastructure there is poor trust in government healthcare facilities especially in tribal areas. $[15,16]$ Various natural calamities damage the road network in the tribal majority areas in the study area causing less access to healthcare services. [17] 
In this context, the study assess the provision of necessary health services for pregnant women in tribal pockets. The study aims to find out the infrastructural adequacy in the health centres of tribal area along with the referral network.

\section{METHODS}

Besides analyzing secondary data, the study adopted cross sectional approach of field survey to understand the healthcare infrastructure in tribal area for provision of maternal healthcare. Both primary and secondary sources of data collection were adopted. Various tools and techniques were used according to the scientific requirement of the study. The following sections specify the locale of the study and source of data collection.

\section{LOCALE OF THE STUDY}

The study was done in the tribal dominated Jaleswar block due to its overall backwardness and challenges in health infrastructure which is assessed from the district healthcare administration. The study area has scope for assessment of a backward block in a relatively developed district like Balasore according to the district administration.

\section{DATA SOURCES}

Modified version of the Parijata Tool developed by United Nations International Children's Fund (UNICEF) is used to assess infrastructure in terms of manpower, availability of drugs and consumables, and norms of clinical practices (ARTH, 2009). For this, health facilities were assessed at CHCHatigarh and $\mathrm{DHH}$-Balasore (Pregnant women from tribal areas of Jaleswar are usually admitted at CHC-Hatigarh for deliveries and $\mathrm{DHH}$-Balasore is the referral centre for obstructed cases of Jaleswar). All the interviews with respondents were audio-recorded by following the protocol with informed consent. The interviews were conducted with the help of them list during the study period (2014-2015). Key administrative informants at PHC/ $\mathrm{CHC}$ and District Health Administration level were interviewed.

\section{DATA ANALYSIS}

Data analysis had been carried out factoring qualitative and quantitative data on the maternal health program. The quantitative data were used to describe various situation in the district health programs. Especially, the scores of parijata tool were gathered and the quality of services in various health facilities were measured with benchmark standards. The qualitative data analysis found different themes in the narrations of care providers, family members, pregnant women and other stakeholders involved in the maternal health program.

Ethical clearance was taken from the committee of the Institute.

\section{RESULTS}

The result section mentions details of infrastructural issues for promotion of maternal health programs which affect the care of pregnant mothers in Balasore district especially in tribal areas. The challenges of the labor room during institutional delivery was assessed with mentioning of specific deficiencies compared with the set standards.

\section{CHALLENGES IN LABOR ROOM DURING INSTITUTIONAL DELIVERY}

The study particularly observed that many harmful procedures are still in practice in government facilities which jeopardize the life of tribal pregnant women. The range of activities is quite big like forceful 'fundal pressure', augmentation of labor to hasten deliveries, poor monitoring of patients post-delivery, and many relevant factors like unhygienic hospital environment, deprived patient nutrition, unscientific management of biomedical wastes in hospital. All these things influence maternal health outcomes even in government facilities. In addition, many beneficial evidence-based practices were not being adopted in the provision of care. This may be due to inadequate training and ineffective monitoring of services and lack of awareness among hospital staff.

For assessment of the quality of perinatal and postnatal services, the Parijata Tool developed by Action Research and Training for Health (ARTH, 2009) in compliance with the guidelines of $\mathrm{WHO}$ has been used in $\mathrm{DHH}$-Balasore and $\mathrm{CHC}$-Hatigarh. CHC-Hatigarh is considered for evaluation as it provides delivery services to pregnant women in the tribal area of Jaleswar. Further, $\mathrm{DHH}$-Balasore acts as a referral centre to $\mathrm{CHC}$-Hatigarh for the treatment of critical care cases relating to maternal health.

The assessment of equipment and supplies is carried out in the labor room for the appropriateness of their use during delivery. The actual availability of items in the labour room was assessed, rather than assessment by questions to the labor room staff. The functionality of the equipment was assessed, and the expiry date of medicine was observed. Timely supply of the equipment and medicines and the status of missing equipment are also gathered from nurse- 
in-charge of the labor room. The assessed scores of $\mathrm{DHH}$ -

Balasore and $\mathrm{CHC}$-Hatigarh are given in Table 1 and 2

respectively.

TABLE 1 ASSESSMENT OF DELIVERY PRACTICES AND AVAILABILITY OF EQUIPMENT AND CONSUMABLES AT DHH-BALASORE

$\begin{array}{lll}\text { PRACTICES } & \text { SCORES } & \text { AWARDED } \\ & & \text { SCORES }\end{array}$

Shaving of pubic hair

$y=0, n=1$

Routine Enema

$y=0, n=1$ CORES

Partograph chart used

$y=1, n=0$

1

Foetal Heart Sound heard during labor

$y=1, n=0$

1

Position of delivery

lithotomy $=0$, sitting $=1 \quad 0$

Augmentation of labor

Episiotomy for prima gravid

$y=0, n=3$

0

$y=0, n=2$

$y=0, n=1$

$y=3, n=0$

$y=0, n=1$

$y=1, n=0$

$y=0, n=1$

mucus sucker=1, electric 1

Equipment used for suction (mucus sucker/ electronic sucker)

sucker $=0$

Initiation of breastfeeding within 1 hour

$\mathrm{n}=0, \mathrm{y}=2$

2

Timing of discharge

$<12 \mathrm{hr}=0,>24 \mathrm{hr}=2$

$\mathrm{y}=1, \mathrm{n}=0$

$y=1, n=0$

1

Hand washing before conducting delivery

$y=2, n=0$

Post-partum checkup in ward

Maximum score possible for this facility

Total score

\% score for practices

$73 \%$

\begin{tabular}{|c|c|c|}
\hline Equipment and supply & & \\
\hline Ambubag kept ready in Labour Room (LR) & $y=1, n=0$ & 1 \\
\hline BP Instrument and Stethoscope ready in LR & $y=1, n=0$ & 1 \\
\hline Washbasin and running water in LR & $y=1, n=0$ & 1 \\
\hline Autoclave present in working condition & $y=1, n=0$ & 1 \\
\hline Labor room clean & $y=1, n=0$ & 0 \\
\hline Labor room condition & clean $=1$, has blood stuck $=0$ & 0 \\
\hline Oxytocin available in LR & $y=1, n=0$ & 1 \\
\hline Staff in Labour Room SBA trained & all=2, half $=1$ & 2 \\
\hline $\begin{array}{l}\text { Doctors (who conduct delivery) oriented on Emergency and } \\
\text { Basic Care (EBC) }\end{array}$ & all $=2$, half $=1$ & 2 \\
\hline IEC material (chart) on evidence-based practices displayed in LR & $y=1, n=0$ & 1 \\
\hline Maximum inputscore for this facility & & 12 \\
\hline Total score & & 10 \\
\hline \% score for practices & & $83 \%$ \\
\hline
\end{tabular}

Source: Modified tools by UNICEF and ARTH, Authors' primary source (2017) 
PRACTICES

Shaving of pubic hair

Routine Enema

Partograph chart used

FHS heard during labor

Position of delivery

Augmentation of labor

Episiotomy for primis

Abdominal pressure

IM oxytocin after delivery

Vaginal packing

Proper drying and wrapping of newborn

Routine suction of all newborn

Equipment used for suction (mucus sucker/ electronic sucker)

Initiation of breastfeeding within 1 hour

Timing of discharge

Sterile gloves used for delivery

Hand washing before conducting delivery

Post-partum checkup in ward

Maximum score possible for this facility

Total score

$\%$ score for practices

Equipment and supply

Ambubag kept ready in LR

BP Instrument and Stethoscope ready in LR

Washbasin and running water in LR

Autoclave present in working condition

Labor room clean

Labor room condition

Oxytocin available in LR

Staff in LR SBA trained

Doctors (who conduct delivery) oriented on EBC

IEC material (chart) on evidence based practices displayed in LR

Maximum inputscore for this facility

Total score

$\%$ score for practices

\section{SCORES}

$\mathrm{y}=0, \mathrm{n}=1$

$y=0, n=1$

$y=1, n=0$

$y=1, n=0$

lithotomy=0, sitting $=1$

$\mathrm{y}=0, \mathrm{n}=3$

$y=0, n=2$

$y=0, n=1$

$y=3, n=0$

$y=0, n=1$

$y=1, n=0$

$\mathrm{y}=0, \mathrm{n}=1$

mucus sucker $=1$, electric sucker $=0 \quad 1$

$\mathrm{n}=0, \mathrm{y}=2$

$<12 \mathrm{hr}=0,>24 \mathrm{hr}=2$

$y=1, n=0$

$y=1, n=0$

$y=2, n=0$

AWARDED

SCORES

1

1

0

0

0

3

0

1

3

1

1

0

2

2

1

2

26

20

$76 \%$

$y=1, n=0$
$y=1, n=0$
$y=1, n=0$
$y=1, n=0$
$y=1, n=0$
clean=1, has blood stuck=0
$y=1, n=0$
$a l l=2$, half $=1$
$y=1, n=0$
$y=0, n=1$

1
1
1
0
0
1
1
2
2
0
12
9
$75 \%$

Source: Modified tools by UNICEF and ARTH, Authors' primary source (2017)

Table 1 and 2 show that DHH-Balasore and $\mathrm{CHC}$-Hatigarh have low scores than the desired minimum, in evidencebased practices for a labor room and regarding availability of equipment and supplies for conducting successful procedures. The DHH-Balasore is trailing by seven points than the required twenty-six points for 'good practices' and is lagging by two points than the required twelve points in 
the availability of essential equipment and supplies. $\mathrm{CHC}$ Hatigarh is lagging by six points than the desired twenty-six points for good practices and is lagging by three points than the desired twelve points in availability for essential facilities. From which, it may be infered that nonadherence to evidence-based practices in the process of labor have a negative impact on the quality of care.

This necessitates effective planning by mutual consultation with district health administrators and medical practitioners. The evidence emerging from the measurement of labor room indicates there is need for improvement of services in $\mathrm{CHC}$-Hatigarh, and $\mathrm{DHH}-$ Balasore.

Findings suggest there is lack of training of medical and paramedical staff in the process of preparedness for good medical care to the mothers. There is lack of orientation of nurses and doctors, and SBA trained nursing staff in the caregiving site. The causes for poor training are erroneous pre-service beliefs and knowledge, lack of support from hospital staff and a general resource crunch. During preservice training, most nurses learn many practices which are not evidence-based (e.g., need for routine episiotomy, need for lithotomy position in delivery), which is reflected in their present handling of patients in the labor room. Suboptimal functioning of labor room and poor facilities in rural $\mathrm{CHC}$ and government hospitals provide little scope for translating knowledge into practices. Unfortunately, these limitations predispose women to risky caesarean section deliveries.

\section{POOR ROAD CONNECTIVITY TO TRIBAL VILLAGES}

Tribal pockets have serious problems in referral and transportation of pregnant women for emergency medical care. The problems get accentuated in the rainy season due to floods and weak surface transport system. The areas surveyed were found to be severely affected for months making it difficult to reach the community facility at $\mathrm{CHC}$ Hatigarh by ambulance services. A Pharmacist working in a flood-prone area narrated:

"The roads constructed under (The) Prime Ministers Gramya Swarojgar Yojna (PMGSY) have helped in reaching the interior tribal pockets during the rainy season which was not otherwise possible a few years ago. As these roads are of good quality and made up of concrete, they withstand the effect of floods. However, these concrete surfaces are not continuous throughout the entire length, connecting remote locations to the health care centres. The villagers sometimes repair minor portions of the 'kaccha road'. Such community initiatives create a new dimension in problem solving, rather than wait for government action."

According to an AYUSH Medical Officer working in the Mobile Health Unit (MHU) for the last five years:

"Prior to concrete roads made by Prime Ministers Gramya Swarojgar Yojna (PMGSY), it was not possible to gain access to tribal villages in the rainy season. During floods, the MHU was parked beside the highway and doctors had to walk up to the village school to attend Village Health and Nutrition Day (VHND) and conduct immunization camps. Due to river embankment, the Jaleswar town is flooded but saved from severe calamities."

\section{NON-AVAILABILITY OF MATERNITY WAITING HOME FOR EMERGENCY MEDICAL SERVICES}

The program implementation plan (PIP) of NRHM-Odisha mentions 'Maa Gruha' as temporary homes for pregnant women ideally located near the hospital where they may wait for safe delivery. This helps in providing emergency services through basic emergency obstetric care (BEmOC). However, no post-partum cases are allowed to stay at 'Maa Gruha'. There are 50 such facilities in the tribal pockets of Odisha which provide care to pregnant women in tribal areas according to NRHM-Odisha.[17] Unfortunately, tribal dominated Jaleswar block was found to have no such provision, thereby depriving tribal women of emergency services. According to health administrators of the block, no alternative support structures exist to redress the problem of inaccessible villages. Ironically, to promote institutional delivery, it is important to reach out to women in an advance stage of pregnancy. This makes facilities like 'Maa Gruha' conspicuous by their absence during the rainy season.

\section{MOBILE MEDICAL TEAM (MMT): OPERATIONAL PROBLEMS}

Mobile medical teams have been commissioned to provide preventive, promotive and curative health care in inaccessible and difficult terrains. In Jaleswar too, MMTs are used to provide support to staff for regular field visits and carry medicines and equipment for care of pregnant women. Also, they provide services at the doorstep to augment hospital services.

The MMT consists of a team comprising of a medical officer (often an AYUSH doctor), a pharmacist, an ANM, an attendant and a driver. The team travels for at least 20 days 
a month to remote villages as per schedule prepared jointly by the block program manager (BPM) and Medical Officer in-charge of $\mathrm{CHC}$-Hatigarh. MMTs not only look after the curative aspects but also contribute to behavior change through IEC activities.

Despite several such provisions, field observations show that MMTs of Jaleswar block were unable to function properly during the rainy season. Health workers in MMT were attached to CHC-Hatigarh which is located on the opposite bank of the river. So, the unit must cross the river across the lone bridge and travel a long distance, thus making the entire operation both expensive and timeconsuming. The flood-affected regions create other problems in access: health workers must walk through muddy water, conduct clinical examinations in open places and manage an unruly crowd. Such circumstances make it difficult to ensure the quality of treatment and care: it is even difficult to ensure the privacy of the women. An AYUSH medical officer recounts the role played by MMTs:

"Until 2008, care was almost denied to tribal areas. Now the MMTs provide essential maternal health services, family planning and other health promoting services for women in remote tribal pockets. Still, there is an unmet need for emergency care. At present, it solves minor pregnancy related problems."

\section{TRANSPORT AND REFERRAL SERVICES}

The transport and referral services in Jaleswar need to be strengthened for better provision of maternal health care given the emergency of situation in some cases. Field assessment shows that many pregnant women still use private transport facilities to reach health centres. During field work, it was found that all three ambulances parked at $\mathrm{CHC}$-Hatigarh were off-road and none provided services in Jaleswar which paralyses the transport to a great extent. The vehicles of course need immediate maintenance, repair, or even replacement with newer ones to serve the tribal community. As a result, much of the need was being met by Janani Express and 108 ambulances.

The 108 ambulance services is a boon which provide effective pre-hospital emergency medical service by (The) Government of Odisha with funding support from MoH\&FW, India. The objective is to provide instant medical care without losing time across rural areas. The service features of the Odisha Emergency Medical Ambulance Service (OEMAS) is designed in such a way that all 108 ambulances are equipped with GPS (Global Positioning System) and MDT (Mobile Data Terminal) and the operation is with a centralized professional call centre. The ambulance is supposed to reach the patient within 20,25, and 35 minutes in urban, semi-urban, rural areas respectively, after getting a requisition from a patient. The mapping and defining of strategic locations for pick-up of patients are spelled out by the ambulance team. The team also specifies the nearest 'catch points' in the case of non-motorable locations for the convenience of pregnant women. As per record of NRHM-Odisha, among transported patients, nearly $23.11 \%$ of the cases consist of pregnant women (NRHM, 2014).

The issue with the 108 is that it does not have drop-back facility, which undermines effectiveness of ambulance services of the women in returning to their home in Jaleswar. As reported by JSY beneficiaries in the community, 108 ambulances do not respond even after repeated calls; there are refusals on account of inaccessibility, long distance to the tribal villages and unavailability of ambulances.

\section{DISCUSSION}

The study found the effectiveness of the maternal health program in the tribal block of Jaleswar is not promising. This is corroborated with the poor maternal health of higher morbidity and mortality among tribal women. The mortality and morbidity figures in Jaleswar are relatively high in comparison to the performance of Balasore district. To achieve the effectiveness in provision of care the study suggest for effectiveness of the ongoing JSY program by enhancing health care infrastructure of the tribal area.

The major issues in implementation of JSY program lies in improvement of geographical and socio-cultural factors in tribal area. This poses special challenges in providing adequate health care infrastructure, connectivity, monitoring through HMIS, capacity building of ASHA workers, and building the trust of the community. These challenges can be addressed by an integrated approach to health care development as suggested by the World Health Organization (2009) in its Social Determinant model. This should also take into consideration governance and policy, culture and social values, health care services at institutional level and influence of family and peer in understanding health of population. However, the study found that JSY has a one-sided focus on biomedically oriented model of care without integrating the cultural 
nuances. The one-fit-all protocols and program adopted by JSY across the country is not suitable for the proper implementation of program in tribal communities like Jaleswar.

Assessment of health centres for infrastructural adequacy is discussed very widely in the study. The study suggests that there is lack of training of medical and paramedical staff in the health care facility. Also, it was found that lack of onsite orientation of nurses and doctors and SBAs is there which hinder proper care. Studies also confirm that there if SBAs do not train properly, it would be difficult to achieve effective maternal health care. [17] This sometimes stems from erroneous pre-service beliefs and knowledge, lack of support from hospital staff and general resource crunch. During pre-service training, most nurses learn many practices which are not evidence-based (e.g., need for routine episiotomy, the need for lithotomy position in delivery), which is reflected in their poor handling of patients in the labor room.

Without optimal functioning of labor room there is less scope for translating knowledge into practice. These limitations can mean risky caesarean section deliveries. An analysis of infrastructural adequacy in Jaleswar by using Parijata Tool shows considerable deficit in equipment, consumables, and essential drugs in health centers of both Jaleswar and Balasore. These deficits, coupled with practices unsupported by evidence-based medicine reflect poor quality of service delivery. Similar findings have been corroborated by others in Odisha. $[17,18]$

Understanding of tribal culture and provision of targeted care is important. The tribal culture and beliefs are not taken into consideration in the program guidelines. A study in Purnia District (Bihar) shows that anaemia and malnutrition is very severe in tribal community and many cultural factors in addition to medical factors are responsible. [19] A study in Odisha also corroborate similar finding on anaemia in tribal community which also affect overall development. Local healers and ethno-medicine play an important part in care of pregnant women in tribal district of Odisha and Jharkhand, India. [20,21,22]

\section{CONCLUSION}

The paper examined how to improve infrastructure to provide optimal maternal healthcare in the study area of Jaleswar. This has been supported by field observation that poor onsite orientation of nurses and doctors, and lack of SBA training, especially to nursing staff results in less quality of services. Sub-optimal functioning of labor room and poor facilities in rural government hospitals provide little scope for treatment of vulnerable tribal population. The analysis by Parijata Tool also brings out the lack of infrastructural facilities in Jaleswar. It shows a considerable deficit in equipments, consumables, and essential drugs in health centres.

Addressing the aforementioned challenges with timely action could help in reduction of maternal deaths in the tribal area. The study raises critical issues about the potential of maternal health program to deliver effective care of pregnant women in tribal dominated areas. The study recommends provision of remedial measures for improving the role and functioning of grass roots workers, integrating indigenous medicine with biomedicine, and revamping health information system to incorporate cultural features, thereby improving its utilization in the study area.

\section{ACKNOWLEDGEMENTS}

University Grants Commission, India for providing funding of the researcher's scholarship. The author has agreed both to be personally accountable for the author's own contributions and to ensure that questions related to the accuracy or integrity of any part of the work, even ones in which the author was not personally involved, are appropriately investigated, resolved, and the resolution documented in the literature.

\section{References}

1. Ministry of Health and Family Welfare, Government of India. Mission Document National Rural Health Mission. New Delhi: Manager of Publication. 2005.

2. World Health Organization. Health in 2015: from MDGs to SDGs. 2015. Retrieved from: http://www.who.int/gho/publications/mdgs-sdgs/en/ (Last Accessed on 27.01.2021)

3. UNICEF. Maternal Health in India. 2013. Retrieved from http://www.unicef.org/india/health.html

4. George A. Persistence of high maternal mortality in Koppal district, Karnataka, India: observed service delivery constraints. Reproductive health matters. 2007 Nov 1;15(30):91-102.

5. Mavalankar DV, Rosenfield A. Maternal mortality in resource-poor settings: policy barriers to care. 
American Journal of Public Health. 2005 Feb;95(2):200-

3.

6. Mavalankar DV, Vora K, Prakasamma M. Achieving millennium development goal 5: is India serious? Bulletin World Health Organization, 86(4), 243-243A.

7. Ramani KV. A management information system to plan and monitor the delivery of health-care services in government hospitals in India. Journal of Health Organization and Management. 2004 Jun 1.

8. Registrar General India, Sample Registration System 2011. Special bulletin on maternal mortality in India 2007-2009. R. K Puram, New Delhi: Vital Statistics Division, Office of RGI. 2011.

9. Evans RG. Incomplete vertical integration: The distinctive structure of the health- care industry. In: Van, Der. Gaag J and Perlman M, (eds). Health, Economics, and Health Economics. Amsterdam: North Holland. 1981.

10. Janovsky K, Cassels A Health policy and systems research: Issues, methods and priorities. In: Janovsky K, Eds. Health Policy and Systems Development: An Agenda for Research. Geneva: World Health Organization, 1996.

11. Roemer MI. National Health Systems of the World, volume 1. New York: Oxford University Press. 1991.

12. Patra S, Singh B, Reddaiah VP. Maternal morbidity during postpartum period in a village of north India: a prospective study. Tropical doctor. 2008 Oct;38(4):2048.

13. Lozano $R$, Wang $H$, Foreman KJ, Rajaratnam JK, Naghavi M, Marcus JR, Dwyer-Lindgren L, Lofgren KT, Phillips D, Atkinson C, Lopez AD. Progress towards Millennium Development Goals 4 and 5 on maternal and child mortality: an updated systematic analysis. The Lancet. 2011 Sep 24;378(9797):1139-65.

14. Ministry of Tribal Affairs, Government of India 2001. Retrieved from: http://tribal.nic.in/WriteReadData/CMS/Documents/2 01306030204039113751 StatewisePTGsList.pdf.

15. Upadhyay RP, Chinnakali P, Odukoya O, Yadav K, Sinha S, Rizwan SA, Daral S, Chellaiyan VG, Silan V. High neonatal mortality rates in rural India: what options to explore?. International Scholarly Research Notices. $2012 ; 2012$

16. Kazembe LN, Mpeketula PM. Quantifying spatial disparities in neonatal mortality using a structured additive regression model. Plos one. 2010 Jun 17;5(6):e 11180.

17. District Administration Balasore 2012. Comprehensive District Annual Plan 2011-12: Balasore District.
Bhubaneswar, Odisha, India: National Bank for Agriculture and Rural Development.

18. Jeffery $P$, Jeffery R. Only when the boat has started sinking: a maternal death in rural north India. Social Science \& Medicine. 2010 Nov 1;71(10):1711-8.

19. Rao TV, Vijay T. Malnutrition and anemia in tribal pediatric population of Purnia district (Bihar). Indian pediatrics. 2006 Feb 1;43(2):181.

20. Balgir RS. Tribal health problems, disease burden and ameliorative challenges in tribal communities with special emphasis on tribes of Orissa. InProceedings of National Symposium on "Tribal Health" 19th-20th October 2006 Oct 20 (pp. 161-176).

21. Das M, Kumar N, Kapoor S, Prasad S. Health issues and health seeking behavior of tribal population in Jharkhand. 2009. USAID

22. Behura NK, \& Mohanty KK 2006. Ethno medicines and ethnic healers in sustainable healthcare services: Case studies from tribal societies of Orissa. In N. K. Behura \& K. K. Mohanty (Eds.) Readings in Social Anthropology.2006 Delhi: Dominant. 